\title{
Front Matter: Volume 9910
}

, "Front Matter: Volume 9910," Proc. SPIE 9910, Observatory Operations: Strategies, Processes, and Systems VI, 991001 (1 August 2016); doi: $10.1117 / 12.2240598$ SPIE Event: SPIE Astronomical Telescopes + Instrumentation, 2016, Edinburgh, SPIE. United Kingdom 


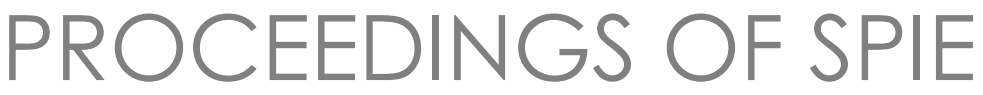

\title{
Observatory Operations: Strategies, Processes, and Systems VI
}

\author{
Alison B. Peck \\ Robert L. Seaman \\ Chris R. Benn \\ Editors
}

27 June - 1 July 2016

Edinburgh, United Kingdom

Sponsored by

SPIE

Cooperating Organizations

American Astronomical Society (United States) • Australian Astronomical Observatory (Australia) - Association of Universities for Research in Astronomy (AURA) •

Canadian Astronomical Society (CASCA) (Canada) • Canadian Space Agency (Canada) • European Astronomical Society (Switzerland) • European Southern Observatory (Germany) • National Radio Astronomy Observatory • Royal Astronomical Society (United Kingdom) • Science \& Technology Facilities Council (United Kingdom)

Published by

SPIE

Volume 9910

Part One of Two Parts

Proceedings of SPIE 0277-786X, V. 9910

SPIE is an international society advancing an interdisciplinary approach to the science and application of light.

Observatory Operations: Strategies, Processes, and Systems VI, edited by Alison B. Peck, Robert L. Seaman, Chris R. Benn, Proc. of SPIE Vol. 9910, $991001 \cdot$ ? 2016 SPIE · CCC code: 0277-786X/16/\$18 · doi: 10.1117/12.2240598 
The papers in this volume were part of the technical conference cited on the cover and title page. Papers were selected and subject to review by the editors and conference program committee. Some conference presentations may not be available for publication. Additional papers and presentation recordings may be available online in the SPIE Digital Library at SPIEDigitallibrary.org.

The papers reflect the work and thoughts of the authors and are published herein as submitted. The publisher is not responsible for the validity of the information or for any outcomes resulting from reliance thereon.

Please use the following format to cite material from these proceedings:

Author(s), "Title of Paper," in Observatory Operations: Strategies, Processes, and Systems VI, edited by Alison B. Peck, Robert L. Seaman, and Chris R. Benn, Proceedings of SPIE Vol. 9910 (SPIE, Bellingham, WA, 2016) Six-digit Article CID Number.

ISSN: 0277-786X

ISSN: 1996-756X (electronic)

ISBN: 9781510601994

ISBN: 9781510602007 (electronic)

Published by

SPIE

P.O. Box 10, Bellingham, Washington 98227-0010 USA

Telephone +1 3606763290 (Pacific Time) · Fax +1 3606471445

SPIE.org

Copyright (C) 2016, Society of Photo-Optical Instrumentation Engineers.

Copying of material in this book for internal or personal use, or for the internal or personal use of specific clients, beyond the fair use provisions granted by the U.S. Copyright Law is authorized by SPIE subject to payment of copying fees. The Transactional Reporting Service base fee for this volume is $\$ 18.00$ per article (or portion thereof), which should be paid directly to the Copyright Clearance Center (CCC), 222 Rosewood Drive, Danvers, MA 01923. Payment may also be made electronically through CCC Online at copyright.com. Other copying for republication, resale, advertising or promotion, or any form of systematic or multiple reproduction of any material in this book is prohibited except with permission in writing from the publisher. The CCC fee code is 0277-786X/16/\$18.00.

Printed in the United States of America.

Publication of record for individual papers is online in the SPIE Digital Library.

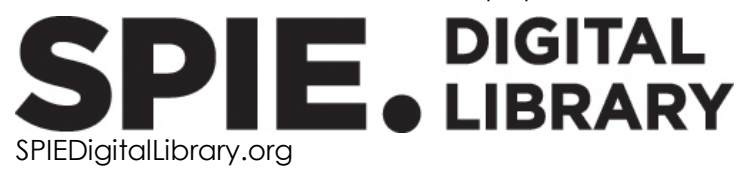

Paper Numbering: Proceedings of SPIE follow an e-First publication model. A unique citation identifier (CID) number is assigned to each article at the time of publication. Utilization of CIDs allows articles to be fully citable as soon as they are published online, and connects the same identifier to all online and print versions of the publication. SPIE uses a six-digit CID article numbering system structured as follows:

- The first four digits correspond to the SPIE volume number.

- The last two digits indicate publication order within the volume using a Base 36 numbering

system employing both numerals and letters. These two-number sets start with 00, 01, 02, 03, 04, 05, 06, 07, 08, 09, OA, OB ... 0Z, followed by 10-1Z, 20-2Z, etc. The CID Number appears on each page of the manuscript. 


\title{
Contents
}

\author{
ix Authors \\ xv Conference Committee \\ xvii Introduction
}

\section{Part One}

SESSION 1 OPERATIONS BENCHMARKING AND METRICS

991002 Operational metrics for the ESO Very Large Telescope: lessons learned and future steps [9910-1]

991003 The impact of science operations on science return at the Very Large Telescope [9910-2]

991004 Improving SALT productivity by using the theory of constraints [9910-3]

991005 A bibliometric analysis of observatory publications for the period 2010-2014 [9910-4]

991006 Observatory bibliographies: a vital resource in operating an observatory [9910-5]

\section{SESSION 2 ARCHIVE OPERATIONS, SURVEYS AND LEGACY DATASETS}

991007 Data products of the ALMA and NRAO archives [9910-6]

991008 Validation of ESO Phase 3 data submissions [9910-7]

991009 Publication of science data products through the ESO archive: lessons learned and future evolution [9910-8]

\section{SESSION 3 VIRTUAL OBSERVATORY}

9910 OA Providing comprehensive and consistent access to astronomical observatory archive data: the NASA archive model [9910-10]

\section{SESSION 4 DATA FLOW AND DATA MANAGEMENT OPERATIONS PROCESSES AND WORKFLOWS}

9910 OB Public surveys at ESO [9910-11]

9910 OC Not letting the perfect be the enemy of the good: steps toward science-ready ALMA images [9910-12] 
9910 OD Science data management for ESO's La Silla Paranal Observatory [9910-13]

SESSION 5 TIME DOMAIN AND TRANSIENT SURVEYS

9910 OE CARMENES: data flow [9910-98]

9910 OF ANTARES: progress towards building a 'broker' of time-domain alerts [9910-14]

99100 DDOTI: the deca-degree optical transient imager [9910-16]

\section{SESSION 6 SITE AND FACILITY OPERATIONS I}

$9910 \mathrm{OH} \quad$ Operations concept for the Square Kilometre Array [9910-17]

99100 Ol The Australian SKA Pathfinder: operations management and user engagement [9910-28]

9910 J Power monitoring and control for large scale projects: SKA, a case study [9910-25]

9910 OL A preliminary operations concept for the ngVLA [9910-18]

$99100 \mathrm{M}$ Centralized operations and maintenance planning at the Atacama Large Millimeter/submillimeter Array (ALMA) [9910-23]

9910 ON STELLA: 10 years of robotic observations on Tenerife [9910-125]

\section{SESSION 7 SITE AND FACILITY OPERATIONS II}

991000 The JCMT as operated by the East Asian Observatory: a brief (but thrilling) history [9910-29]

9910 OP Precipitable Water Vapour at the Canarian Observatories (Teide and Roque de los Muchachos) from routine GPS [9910-33]

$99100 Q$ CARMENES-NIR channel spectrograph: how to achieve the full AIV at system level of a cryo-instrument in nine months [9910-32]

9910 OR Response to major earthquakes affecting Gemini twins [9910-22]

9910 OT Worth its SALT: four years of full science operations with the Southern African Large Telescope [9910-26]

9910 OV A collimated beam projector for precise telescopes calibration [9910-27]

9910 OW SOAR telescope operation in the LSST era: real-time follow-up on large scales [9910-30]

9910 0X Organizational transformation to improve operational efficiency at Gemini South [9910-31] 
9910 OY Sharing the skies: the Gemini Observatory international time allocation process [9910-109]

$99100 Z$ Getting NUSTAR on target: predicting mast motion [9910-35]

991011 Feature-based telescope scheduler [9910-37]

991012 Ongoing evolution of proposal reviews in the Spitzer warm mission [9910-38]

\section{SESSION 9 PROGRAM AND OBSERVATION SCHEDULING II}

991013 The LSST Scheduler from design to construction [9910-39]

991014 Survey strategy optimization for the Atacama Cosmology Telescope [9910-41]

991015 SNR-based queve observations at CFHT [9910-42]

991016 Pandeia: a multi-mission exposure time calculator for JWST and WFIRST [9910-44]

991018 Hinode/EIS science planning and operations tools [9910-46]

$99101 \mathrm{~A} \quad$ An optical to IR sky brightness model for the LSST [9910-48]

9910 1B Measurements of airglow on Maunakea at Gemini Observatory [9910-49]

\section{SESSION 10 OPERATIONS AND DATA QUALITY CONTROL}

9910 1C Two years of LCOGT operations: the challenges of a global observatory [9910-50]

$99101 \mathrm{D}$ The dark energy survey and operations: years 1 to 3 [9910-51]

$99101 \mathrm{E} \quad$ Lessons from and methods for surveying large areas with the Hubble Space Telescope [9910-52]

9910 IF Spectral calibration for the Maunakea Spectroscopic Explorer: challenges and solutions [9910-54]

$99101 \mathrm{G}$ Calibration development strategies for the Daniel K. Inouye Solar Telescope (DKIST) data center [9910-55]

$99101 \mathrm{H} \quad$ ALMA quality assurance: concepts, procedures, and tools [9910-56]

$991011 \quad$ Data mining spacecraft telemetry: towards generic solutions to automatic health monitoring and status characterisation [9910-58] 


\section{Part Two}

SESSION 11 SCIENCE OPERATIONS PROCESSES AND WORKFLOWS I

$99101 \mathrm{~J}$ The evolution of observing modes at ESO telescopes [9910-59]

$99101 \mathrm{~K} \quad$ New facilities, new challenges: the telescope and instrument operators evolution at ESO [9910-60]

SESSION 12 SCIENCE OPERATIONS PROCESSES AND WORKFLOWS II

$99101 \mathrm{M}$ Science operations at Gemini Observatory [9910-62]

$991010 \quad$ Planning JWST NIRSpec MSA spectroscopy using NIRCam pre-images [9910-64]

$99101 \mathrm{P} \quad$ Moving toward queve operations at the Large Binocular Telescope Observatory [9910-65]

$99101 Q \quad$ The 4MOST Operations System [9910-63]

9910 is Through thick and thin: quantitative classification of photometric observing conditions on Paranal [9910-68]

$99101 \mathrm{~A} \quad$ A daily task manager for Paranal Science Operations [9910-69]

$99101 \mathrm{U}$ Delivering data reduction pipelines to science users [9910-71]

POSTER SESSION

9910 IV ASTRI SST-2M archive system: a prototype for the Cherenkov Telescope Array [9910-72]

$99101 \mathrm{~W} \quad$ Usefulness and dangers of relying on grant acknowledgments in an observatory bibliography [9910-73]

$99101 \mathrm{X}$ Gemini base facility operations environmental monitoring: key systems and tools for the remote operator [9910-74]

$99101 Y \quad$ Approximations of the synoptic spectra of atmospheric turbulence by sums of spectra of coherent structures [9910-75]

991012 ALMA Array Operations Group process overview [9910-76]

991020 System-dependent earthquake inspection procedures at Paranal Observatory [9910-77]

991021 SystMon: a data visualization tool for the analysis of telemetry data [9910-78]

991022 Obsolescence of electronics at the VLT [9910-80]

991023 Operation of AST3 telescope and site testing at Dome A, Antarctica [9910-81]

991024 Operations of the laser traffic control system in Paranal [9910-82] 
991025

991026

991027

991028

991029

$99102 \mathrm{~A}$

$99102 B$

$99102 \mathrm{E}$

$99102 F$

$99102 G$

$99102 \mathrm{H}$

99102

$99102 \mathrm{~J}$

$99102 \mathrm{~L}$

$99102 \mathrm{M}$

991020

$99102 P$

991025

$99102 \mathrm{~T}$

$99102 U$

$99102 W$
Planning your JWST/NIRSpec observation: pre-imaging and source catalogue [9910-83]

Sun avoidance strategies at the Large Millimeter Telescope [9910-84]

Observing with FIFI-LS on SOFIA: time estimates and strategies to use a field imaging spectrometer on an airborne observatory [9910-85]

High precision tracking method for solar telescopes [9910-86]

ESO Phase 3 automatic data validation: groovy-based tool to assure the compliance of the reduced data with the Science Data Product Standard [9910-87]

Automated scheduler improvements and generalizations for the Automated Planet Finder [9910-88]

Trends and developments in VLT data papers as seen through telbib [9910-89]

Data reduction pipelines for the Keck Observatory Archive [9910-93]

E-ELT HIRES the high resolution spectrograph for the E-ELT: integrated data flow system [9910-94]

Aircraft avoidance for laser propagation at the Large Binocular Telescope Observatory: life under a busy airspace [9910-95]

Genetically optimizing weather predictions [9910-96]

Detailed design of a deployable tertiary mirror for the Keck I telescope [9910-97]

DAG telescope site studies and infrastructure for possible international co-operations [9910-99]

Education and public engagement in observatory operations [9910-101]

Monitoring the performance of the Southern African Large Telescope [9910-102]

Quality control and data flow operations of SPHERE [9910-104]

Development of an automated data acquisition and processing pipeline using multiple telescopes for observing transient phenomena [9910-106]

Model-based fault detection and diagnosis in ALMA subsystems [9910-110]

Investigating the effect of atmospheric turbulence on mid-IR data quality with VISIR [9910-111]

DAG: a new observatory and a prospective observing site for other potential telescopes [9910-1 13]

The MIRI Medium Resolution Spectrometer calibration pipeline [9910-117] 
$99102 X$ Linear and Angolar Moment of a general spherical TEM and DEM beam radio wave detection with a quadratic order system processor in state of art technology

implementation: a three axis sensors array quadratic order correlator for the $21 \mathrm{~cm}$ radiation radio detection coming from Early Universe [9910-118]

$99102 Y \quad$ Evolution of operations for the Survey Telescope at Paranal [9910-119]

$99102 Z$ PHOENIX: the production line for science data products at ESO [9910-120]

991030 ESO science data product standard for 1D spectral products [9910-121]

991031 Building a pipeline of talent for operating radio observatories [9910-122]

991032 Training telescope operators and support astronomers at Paranal [9910-123]

991033 SPOT: an optimization software for dynamic observation programming [9910-126] 


\title{
Authors
}

Numbers in the index correspond to the last two digits of the six-digit citation identifier (CID) article numbering system used in Proceedings of SPIE. The first four digits reflect the volume number. Base 36 numbering is employed for the last two digits and indicates the order of articles within the volume. Numbers start with 00, 01, 02, 03, 04, 05, 06, 07, 08, 09, OA, OB...0Z, followed by 10-1Z, 20-2Z, etc.

\author{
Abbott, T. M. C., OV, 1D \\ Abril, M., $0 Q$ \\ Accomazzi, Alberto, OA \\ Adamson, Andy, 1M \\ Adkins, Sean, 21 \\ Akiyama, E., $1 \mathrm{H}$ \\ Alarcon, Hector, 12 \\ Aleksić, J., 1D \\ Alis, Sinan, 2J \\ Allam, S., 1D \\ Aloisi, Alessandra, OA \\ Alonso, D., 14 \\ Alonso-Floriano, F. J., OE \\ Amado, P. J., OE, OQ \\ Amico, P. 24 \\ Anderson, Joe, 32 \\ Ángeles, Fernando, OG \\ Angeli, George Z., 1 A \\ Annis, J., 1D \\ Antonelli, Lucio Angelo, $1 \mathrm{~V}$ \\ Armus, Lee, 12 \\ Arnaboldi, Magda, 08, 09, 0B, 29, 30 \\ Arriagada, Gustavo, $1 \mathrm{X}$ \\ Arsenault, R., 24 \\ Ashley, Michael C. B., 23 \\ Asmus, Daniel, 2T \\ Astier, Joseph, 1P \\ Axelrod, Tim, OF \\ Azzollini, R., 2W \\ Bailey, J., 2W \\ Balbinot, E., 1D \\ Ball, Jesse, 1X \\ Barbosa, Domingos, OJ \\ Barker, Elizabeth A., 16 \\ Barraca, João Paulo, OJ \\ Barrios, Emilio, $1 \mathrm{Z}$ \\ Basa, Stéphane, OG \\ Baude, Laurence, 33 \\ Baumer, M., ID \\ Beard, S., 2W \\ Beaufore, L., 1D \\ Becerril, S., OQ \\ Bechtol, K., 1D \\ Beck, Tracy L., 10, 25 \\ Beckmann, Siman, 27 \\ Bell, Graham S., 00 \\ Bernstein, G., 1D \\ Berriman, Graham B., OA, 2E \\ Berukoff, Steven J., 1G
}

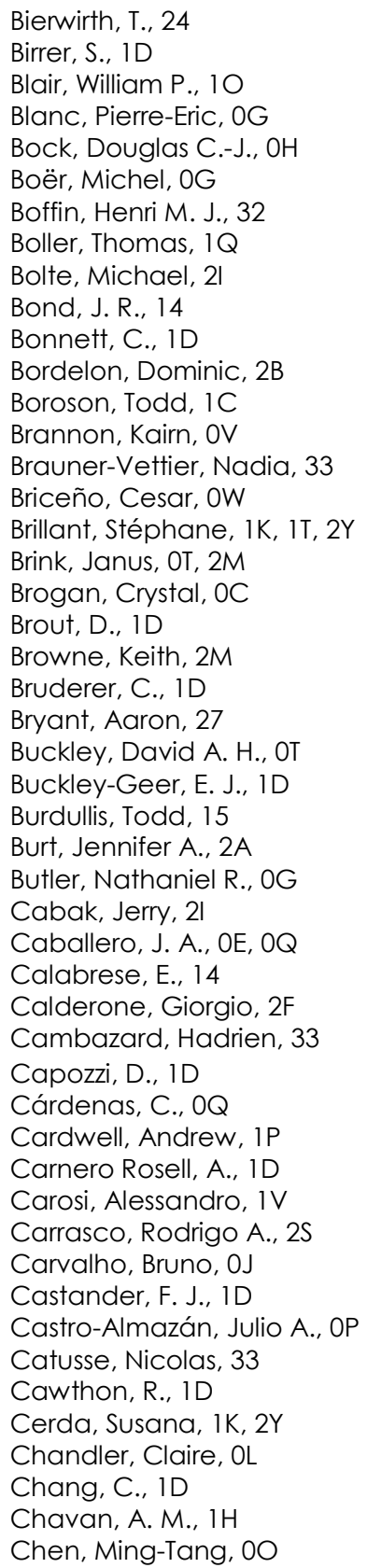


Chiappini, Cristina, 1Q

Choi, S. K., 14

Christou, Julian C., 2G

Chrysostomou, Antonio, $\mathrm{OH}$

Cimmino, Rosario F., 2X

Claver, Charles F., OV, 1 A

Clerc, Nicolas, 1Q

Clerkin, L., ID

Coccato, Lodovico, $2 \mathrm{Z}$

Coetzee, Johannes C., 04, OT, 2M

Cohen, R., 2E

Colditz, Sebastian, 27

Collins, Paul, $1 X$

Colomé, J., $\mathrm{OE}$

Colson, A., 2E

Connolly, Andrew J., 1 A

Cook, Kem, IA

Cordova, Martin, IX

Cortés-Contreras, M., OE

Costa, Alessandro, IV

Coughlin, Michael, OV, IA

Covarrubias, R., 1D

Cowley, David, 2 l

Crabtree, Dennis R., 05

Craig, William W., $\mathrm{OZ}$

Crane, Megan, 12

Crawford, Steven M., 04, 0T, 2M

Cristiani, Stefano, 2F

Crowley, K. T., 14

Cuhna, C., ID

Cuillandre, Jean-Charles, 15

Cupani, Guido, 2F

da Costa, L., 1D

Dahler, Mike, 21

D'Andrea, C., 1D

Daniel, Scott, $1 \mathrm{~A}$

Das, R., 1D

Davis, C., 1D

Davis, Gary R., $\mathrm{OH}$

De Bernardis, F., 14

De Bruyn, David, $2 M$

de Diego, Jose A., OG

de Guindos, E., OE

de Jong, Roelof S., 1Q

de Juan, E., OE

De Meester, W., 11

De Ridder, J., 11

de Wit, Willem-Jan, 32

Deich, William T. S., 2A, 21

Delgado, Francisco, 13

Delmotte, Nausicaa A. R., 08, 09, 0B, 29, 30

Dempsey, Jessica T., 00

Depagne, Éric, 04, 0T

Desai, Vandana R., OA

Devlin, M., 14

Devost, Daniel, 15

Di Marcantonio, Paolo, $2 F$

Dicken, D., 2W

Diehl, H. T., 1D

Dietrich, J., 1D
Dodd, Suzanne R., 12

D'Odorico, Valentina, 2F

Doherty, Peter, OV

Donaldson, Thomas, OA

Donovan Meyer, Jennifer, OC

Dower, Theresa, OA

Drlica-Wagner, A., ID

Duhoux, P., 24

Dunkley, J., 14

Dwelly, Tom, 1Q

Earl, Nicholas M., 16

Ebert, Rick, OA

Edwards, Michelle L., IP

Eifler, T. F., 1D

Elias, Jonathan $\mathrm{H} .$, OW

Elliott, A., 1D

Etherington, J., 1D

Evans, D. J., 11

Fabbiano, Giuseppina, OA

Farah, Alejandro S., OG

Fausti Neto, A., 1D

Feng, Song, 28

Fernández, M. G., 1D

Ferro, I., OQ

Figueroa, Liliana, OG

Fischer, Christian, 27

Fisher-Levine, Merlin, OV

Flagey, Nicolas, IF

Flaugher, B. L., 1D

Forchì, V., 29

Forster, Karl, $\mathrm{OZ}$

Freudling, Wolfram, $1 \mathrm{U}$

Friberg, Per, 00

Frieman, J., 1D

Fuentes, Javier, $1 \mathrm{X}$

Fumi, Fabio, 27

Furlanetto, C., 1D

Gabor, Pavel, 2L

Gadotti, Dimitri A., 32

Gairing, Stefan, OM

Gallardo, P. A., 14

Gallozzi, Stefano, IV

Gangkofner, D., 1D

García-Lorenzo, Begoña, OP

García-Marín, M., 2W

García-Piquer, Á., OE

Geers, V., 2W

Gehrels, Neil A., OG

Geis, Norbert, 27

Gelino, C. R., 2E

Gerdes, D. W., 1D

Gesa, L., OE

Gilbert, Karoline, 16, 10

Girard, Julien H. V., 2O, 32

Glasse, A., 2W

Glauser, A., 2W

Goldstein, D. A., 1D

Gómez Maqueo Chew, Yilen, OG

Gordon, K., 2W

Grabowski, K., 1D 
Granzer, Thomas, ON

Grefenstette, Brian W., OZ

Groom, Steve L., OA

Grothkopf, Uta, 03, 2B

Gruendl, R., 1D

Guàrdia, J., OE

Güçsav, B. Bülent, 2U

Gueguen, Alain, 1Q

Guerra, Juan Carlos, 21

Guniat, Serge, OM

Guo, Jingjing, 28

Gupta, R. R., 1D

Haddad, Juan-Pablo, 22

Hagen, H.-J., OE

Hainaut, O., $1 \mathrm{~J}$

Hamilton, S., 1D

Hanley, Christopher, 16

Hanuschik, Reinhard, 1S, $2 Z$

Harrison, Fiona A., $\mathrm{OZ}$

Harvey-Smith, Lisa, Ol

Hasselfield, M., 14

Hau, G., $1 \mathrm{~J}$

Hays, Tony, $1 G$

Head, H., ID

Helmling, J., OE

Helsby, J., 1D

Henderson, S. W., 14

Henning, Thomas, 27

Hernández Castaño, L., OE

Hernandez, Octavio, OM

Hettlage, Christian, 04, 2M

Hibbard, John, OC, OL

Hilker, Michael, 02, OB

Hilton, M., 14

Hlozek, R., 14

Ho, Paul T. P., 00

Ho, S. P., 14

Holden, Bradford P., 2A

Hollowood, D., 1D

Hönle, Rainer, 27

Honscheid, K., 1D

$\mathrm{Hu}$, Keliang, 23

$\mathrm{Hu}, \mathrm{Yi}, 23$

Hüdepohl, Gerhard, 22

Huffenberger, K., 14

Hulme, Stephen, 2M

Hummel, Christian, 2T

Hummel, Wolfgang, 20

Hussain, Gaitee, OB

Huygen, R., 11

Imel, David, OA

Indebetouw, Remy, OC

Ingraham, Patrick, OV

Iserlohe, Christof, 27

Ivezić, Željko, IA

Ivison, Rob, OB

James, D., 1D

Jenness, Tim, OF

Ji, Kanfan, 28

Johnson, M. W. G., ID
Johnson, M., 1D

Jones, R. Lynne, 1 A

Jouvel, S., ID

Justtanont, K., 2W

Kacprzac, T., 1D

Karakla, Diane M., 16, 10

Kassin, Susan A., 10

Kaufer, A., 02, 03

Käufl, Hans-Ulrich, 2T

Kececioglu, John, OF

Kent, S., 1D

Kepley, Amanda A., OC

Kerber, Florian, 1S, 2T

Kern, Jeff, OL

Keskin, Onur, 2J, 2U

Kessler, R., 1D

Keyes, Charles D., 10

Kim, A., 1D

Klaassen, $\mathrm{P}$., 2W

Klein, Randolf, 27

Klotz, Alain, OG

Kniazev, Alexei, 04, 0T

Koen, Thea, OT

Kong, M., 2E

Koopman, B. J., 14

Korte-Stapff, M., 11

Kosowsky, A., 14

Kotze, Paul, 04, 0T, 2M

Krabbe, Alfred, 27

Krause, E., 1D

Krawiec, C. I., 1D

Kremin, A., 1D

Kron, R., 1D

Kuhlmann, S., 1D

Kuntschner, H., 24

Kuropatkin, N., 1D

Kurowski, R., $1 \mathrm{H}$

Kürster, M., OE

Kutyrev, Alexander S., OG

La Fuente, Carlos, $2 Y$

Labiano, A., 2W

Lacy, Mark, 07

Lagrange, Anne-Marie, 33

Lahav, O., ID

Lahuis, F., 2W

Laidler, Victoria G., 16

Laine, Seppo, 12

Laity, A. C., 2E

Landry, Walter, OA

Lasker, J., ID

Law, D., 2W

Le Roux, Gerhard, 0J

Lee, William H., OG

Lefebvre, Michael, 2G

Leggett, Sandy, $1 M$

Leibundgut, Bruno, 03, OB

Lemaire, Pierre, 33

Lewis, Hilton, 21

Li, T. S., 1D

Lin, Jiaben, 28 
Lombardi, Saverio, $1 \mathrm{~V}$

Looney, Leslie W., 27

López del Fresno, M., OE

Lopez, Bernhard, OM

López-Santiago, J., OE

Lovis, T., 14

Lucarelli, Fabrizio, $1 \mathrm{~V}$

Lucuix, Christian, 22

Lukin, Vladimir P., IY

Lundin, Lars, 20

Lupton, Robert, OV

Luque, E., 1D

Ma, Bin, 23

Maartens, Deneys, 2M

Maccrann, N., ID

MacKenty, John W., IE

Madec, P.-Y., 24

Mader, J. A., 2E

Madhavacheril, M. S., 14

Madonna, Alberto, IV

Madsen, Kristin K., OZ

Mahoney, Billy, 15

Maia, Dalmiro, OJ

Mainieri, $\vee ., 02$

Maltes, Diego, OX

Manset, Nadine, 15

Marang, Fred, OT

March, M., ID

Marconi, Alessandro, 2F

Margheim, Steven J., OY

Markwardt, Craig B., OZ

Marshall, J., ID

Marteau, S., 02, $1 \mathrm{~J}$

Martinez, J., 11

Mascetti, Laura, 08, 09, 0B, 29, 30

Mason, Brian, $0 \mathrm{C}$

Mastropietro, Michele, IV

Masuda, Neal, IX

Matheson, Thomas, OF

Mayo, Louis, 2L

Mazzerella, Joseph M., OA

McConnachie, Alan, IF

McGlynn, Thomas, OA

McKinnon, Mark, OL

McMahon, J., 14

Meakins, Silvia, 2B

Melchior, P., 1D

Menanteau, F., ID

Mérand, Antoine, 1K

Merloni, Andrea, 1Q

Meza, Francisco, $1 \mathrm{X}$

Micol, Alberto, 08, 09, 0B, 29, 30

Mieske, Steffen, 02, 03, 0B, 1J, 1K, 1T, 2Y

Mignot, Shan, $1 \mathrm{~F}$

Milli, Julien, 20

Mirabet, E., $0 Q$

Miszalski, Brent, 04, OT

Miyasaka, Hiromasa, $\mathrm{OZ}$

Momcheva, Ivelina, IE

Mondrik, N. P., ID
Mondrik, Nicholas, OV

Montes, D., OE

Montes, Vanessa, OR

Morales MUñoz, R., OE, OQ

Moreno, Cristian, OR

Morganson, E. P., ID

Morrison, J., 2W

Moullet, Arielle, 0C

Moutou, Claire, 15

Mudd, D., ID

Müller, M., 2W

Muñoz-Tuñón, Casiana, OP

Murowinski, Rick, IF

Mysore, S., 02

Nadolski, A., 1D

Naess, S., 14

Nagasawa, D. Q., 1D

Naghib, Elahesadat, 11

Narayan, Gautham, OF

Natarajan, Swaminathan, OJ

Nati, F., 14

Neilsen, E., 1D

Nelson, Jerry, 21

Neureiter, Bianca, is

Newburgh, L., 14

Niemack, M. D., 14

Nord, B., 1D

Noriega-Crespo, Alberto, 16

Nosov, Eugene V., IY

Nosov, Viktor $V_{\text {., }} 1 Y$

Nugent, P., 1D

O'Donoghue, Darragh E., 04

Ogando, R., 1D

Old, L., 1D

Ortiz, José, $2 \mathrm{~S}$

Osorio, Juan, 20, 21

Page, L. A., 14

Palmese, A., 1D

Park, Sam, 2

Parsons, Harriet, 00

Patat, F., 02, 03, $1 \mathrm{~J}$

Pavlov, A., OE

Peck, Michael, 2l

Pena, Eduardo, 21

Peña-Guerrero, Maria A., 10

Pérez, D., OQ

Perez, Gabriel, OR

Pérez-Jordán, Gabriel, OP

Perley, Margaret, OL

Petravick, D., 1D

Petr-Gotzens, Monika, OB

Petry, Catherine, IA

Pevunova, Olga, OA

Phillips, Drew, 21

Pickering, Timothy E., 16

Pino Pavez, Andres, 1K, 1T, 2Y, 32

Plazas, A. A., 1D

Poglitsch, Albrecht, 27

Pollard, Mike, 2l

Pomante, Emanuele, $2 \mathrm{~F}$ 
Pontoppidan, Klaus M., 16

Potter, S. B., 2H

Power, Jennifer, IP

Primas, F., 02

Prochaska, J. Xavier, 2

Pruemm, M., 24

Pueyo, Laurent, 16

Pujol, A., 1D

Queiroz, A. B. A., 1D

Querel, Richard R., is

Quirós, Fernando, OG

Quirrenbach, A., $\mathrm{OE}, \mathrm{OQ}$

Raab, Walfried, 27

Rabe, Paul, 04, 2M

Racine, René, 15

Rahmer, Gustavo, 2G

Rainnie, Jonn A., 18

Ramirez, A., 20

Ramón, A., $0 Q$

Rana, Vikram R., $0 Z$

Randall, S., $1 \mathrm{H}$

Randolph, Bill, 2 I

Ratliff, Chris, 2l

Ravindranath, Swara, 16

Reardon, Kevin, $1 G$

Rebell, Felix, 27

Rebull, L. M., 12

Regibo, S., 11

Reid, I. N., 10

Reil, K., 1D

Reiners, A., OE, OQ

Rejkuba, Marina, 02, 03, 0B, 1 J, 24

Retzlaff, Joerg, 08, 09, 0B, 29, 30

Reuter, Michael A., 13, 1 A

Reyes, Claudia, $2 Y$

Reyes-Ruíz, Maurico, OG

Ribas, I., OE, OQ

Ridgway, Stephen, OF

Rieke, G., 2W

Rippa, Mathew, OR

Rivas, Leonel, IT

Robberto, Massimo, 16

Rodrigues, Myriam, $1 \mathrm{~T}$

Rogers, Rolando, OR, OX

Romaniello, Martino, 03, OB, OD, $1 \mathrm{U}$

Romano, Francesco, $2 X$

Román-Zúñiga, Carlos G., OG

Romer, A. K., 1D

Romero, Cristian Marcelo, 1K, 1T, 2Y

Romero, Ignacio, OP

Romero-Colmenero, Encarni, 04, 0T, 2H, 2M

Roodman, A., ID

Rooney, P., 1D

Rosenfeld, R., 1D

Roth, Katherine C., $1 \mathrm{~B}$

Rots, Arnold, 06, IW

Royer, P., 11

Rubini, Pascal, 33

Ruíz-Díaz-Soto, Jaime, OG

Rutten, René, $1 \mathrm{M}$
Saha, Abhijit, OF

Sako, M., ID

Salatino, M., 14

Salvador, A. I., 1D

Sánchez Álvaro, E., 1D

Sánchez, C., 1D

Sánchez-Carrasco, M. A., OQ

Sandford, Dale, 2l

Santiago, B. X., 1D

Santos, P., 24

Savant, Vaibhav, 2P

Saviane, I., $1 \mathrm{~J}$

Scheidegger, Carlos, OF

Schillaci, A., 14

Schloerb, F. Peter, 26

Schmitt, B. L., 14

Schooneveld, A., 1D

Schroeder, Anja, OT

Schubnell, M., 1D

Seaman, Robert, OF

Seca, Luis, OJ

Sehgal, N., 14

Seifert, W., OE, OQ

Serio, Andrew, $1 \mathrm{X}$

Shang, Zhaohui, 23

Sheldon, E., 1D

Sienkiewicz, Mark J., 16

Sievers, J. L., 14

Silbermann, Nancy A., 12

Simon, S. M., 14

Slocum, Christine, 16

Smale, Alan, OA

Smette, Alain, 1K, 2T

Smirnova, Olesja, 1B

Smith, A., 1D

Smith, Adam, 1B

Smith, David R., 26

Smith, Niall, $2 P$

Smith, R. C., 1D

Snodgrass, Richard, OF

Soares-Santos, M., ID

Sobreira, F., ID

Soderblom, D. R., 10

Solano, E., OE

Sontag, Christopher D., 16

Souccar, Kamal, 26

Soumagnac, M., ID

Speiss, Daniel J., $1 G$

Spergel, D. N., 14

Spinka, H., ID

Staats, Kai, $2 \mathrm{H}$

Staggs, S. T., 14

Stauffer, John, 12

Stephan, Christian, 21

Stephens, Andrew, 1B

Sterzik, Michael, 02, 03, 2B

Stevens, J. R., 14

Storrie-Lombardi, Lisa J., 12

Strassmeier, Klaus G., ON

Strydom, Ockert, OT, 2M 
Stubbs, Christopher, 0V, 11, 1A

Suarez Sola, Igor, 1P

Summers, Doug, 1P, 24

Swain, M., 2E

Swart, Paul, 0J

Swett, Hector, $1 \mathrm{X}$

Szeifert, Thomas, OB

Szeto, Kei, IF

Tacconi-Garman, L. E., 02, 03, $1 \mathrm{~J}$

Taljaard, Cornelius, $\mathrm{OH}$

Tanne, S. L., 1H

Taylor, Clark, OF

Taylor, Julian, 2T

Teplitz, Harry I., OA

Thierry, Pierre, OG

Tie, S. S., 1D

Tinoco, Silvio, OG

Toeniskoetter, Jackson, OF

Torgaev, Andrey V., IY

Tran, H. D., 2E

Trifiletti, Alessandro, 2X

Trinh, Christopher, 27

Tripsas, Alex, 2

Tristram, Konrad, 2T

Troja, Eleonora, OG

Tucker, D., 1D

Úbeda, Leonardo, 10, 25

Väisänen, Petri, 04, 0T, 2M

Valenzuela, Javier, 21

van Ardenne, Arnold, 0J

van den Ancker, Mario E., 2T

van der Hoeven, Michiel, OR, OX

van Engelen, A., 14

Van Wyk, Veronica, OT

Vandenbussche, B., 11, 2W

Vanderbei, Robert J., 11

Varela, Antonia, OP

Vavagiakis, E. M., 14

Veillet, Christian, 1P

Vieira, Jorge, 0J

Vigan, Arthur, 20

Vikram, V., 1D

Vila Vilaro, B., $1 \mathrm{H}$

Villard, E., 1H

Vinther, Jakob, 2T

Vivas, K., 1D

Volgenau, Nikolaus, 1C

Wahhaj, Zahed, 20

Walcher, Jakob C., 1Q

Walker, A. R., 1D

Wallace, Gary, 26

Walsh, Shane, 1P

Walther, Craig, 00

Wang, Bingxiang, 28

Wang, Lifan, 23

Wang, Zhe, OF

Ward, Jim, 2

Watson, Alan M., OG

Watson, Fraser T., IG

Weber, Michael, ON
Welch, Eric, OF

Wester, W., 1D

White, Richard L., OA

Whyborn, Nicholas D., OM

Wiant, Scott, $1 \mathrm{G}$

Wiesner, M., 1D

Wilcox, H., 1D

Williams, P., 1D

Williams, Theodore B., OT

Wingate, Lory M., 31

Winkelman, Sherry, 06, 1W

Wold, Truman, 2l

Wolff, Burkhard, 2T

Wollack, E. J., 14

Wright, G., 2W

Xin, Bo, $1 \mathrm{~A}$

Yang, Shuo, OF

Yang, Yunfei, 28

Yanny, B., 1D

Yerli, Sinan K., 2J, 2U

Yeşilyaprak, Cahit, 2J, 2U

Yoachim, Peter, 1A

Yuan, Xiangyan, 23

Zaidi, Tayeb, OF

Zampieri, S., 29

Zaritsky, Dennis, 2L

Zechmeister, M., OE

Zeng, Zhen, 28

Zenteno, A., 1D

Zhang, Y., ID

Zhang, Z., ID 


\section{Conference Committee}

Symposium Chairs

Colin Cunningham, UK Astronomy Technology Centre

(United Kingdom)

Masanori lye, National Astronomical Observatory of Japan (Japan)

Symposium Co-chairs

Allison A. Barto, Ball Aerospace \& Technologies Corporation

(United States)

Suzanne K. Ramsay, European Southern Observatory (Germany)

Conference Chairs

Alison B. Peck, National Radio Astronomy Observatory (United States)

Robert L. Seaman, The University of Arizona (United States)

Chris R. Benn, Isaac Newton Group of Telescopes (Spain)

Conference Program Committee

David S. Adler, Space Telescope Science Institute (United States)

Lori E. Allen, National Optical Astronomy Observatory (United States)

Todd Boroson, Las Cumbres Observatory Global Telescope Network

(United States)

Dennis R. Crabtree, National Research Council Canada (Canada)

Suzanne R. Dodd, Jet Propulsion Laboratory (United States)

Andreas Kaufer, European Southern Observatory (Chile)

Nicole M. Radziwill, James Madison University (United States)

Arnold H. Rots, Harvard-Smithsonian Center for Astrophysics

(United States)

Christian Veillet, Large Binocular Telescope Observatory

(United States)

\section{Session Chairs}

1 Operations Benchmarking and Metrics

Alison B. Peck, National Radio Astronomy Observatory (United States)

2 Archive Operations, Surveys and Legacy Datasets

Dennis R. Crabtree, NRC - Herzberg Astronomy \& Astrophysics

(Canada) 
3 Virtual Observatory

Dennis R. Crabtree, NRC - Herzberg Astronomy \& Astrophysics

(Canada)

4 Data Flow and Data Management Operations Processes and Workflows

Todd Boroson, Las Cumbres Observatory Global Telescope Network (United States)

5 Time Domain and Transient Surveys

Todd Boroson, Las Cumbres Observatory Global Telescope Network (United States)

6 Site and Facility Operations I

Chris R. Benn, Isaac Newton Group of Telescopes (Spain)

7 Site and Facility Operations II

Dennis R. Crabtree, NRC - Herzberg Astronomy \& Astrophysics

(Canada)

8 Program and Observation Scheduling I

Suzanne R. Dodd, Jet Propulsion Laboratory (United States)

9 Program and Observation Scheduling II

Todd Boroson, Las Cumbres Observatory Global Telescope Network (United States)

Alison B. Peck, National Radio Astronomy Observatory (United States)

10 Operations and Data Quality Control

Andreas Kaufer, European Southern Observatory (Chile)

11 Science Operations Processes and Workflows I

Chris R. Benn, Isaac Newton Group of Telescopes (Spain)

12 Science Operations Processes and Workflows II

Christian Veillet, Large Binocular Telescope Observatory (United States)

Alison B. Peck, National Radio Astronomy Observatory (United States) 


\section{Introduction}

This conference provided a forum for discussion of a broad range of issues relevant to the operation of ground-based and space observatories, including observing/support models, calibration, data-reduction/archiving, quality control, engineering and infrastructure, fault-handling, productivity, and staffing.

While every ground and space observatory has its own individual and unique characteristics, each shares with the others a common need to execute technical and science operations in the most efficient and cost-effective way possible. This need is driven by the upward pressure from users for more services and capabilities in opposition to the downward pressure by funding agencies to contain or reduce costs. Tension at the interface between users and funders is particularly acute in this time of global economic turmoil. At the same time the technical and logistical challenges are growing with the systems and network complexity of new observing modes, coordinated multi-facility and multimessenger observing campaigns, fully or partially robotic facilities, integrated instrument pipelines and science archives, and the need to integrate more complex cyber-infrastructure such as the Grid and the Virtual Observatory. The subtle intricacies and mega-scales of new instrumentation demand correspondingly creative operations modalities.

Building on previous successful conferences, we gathered to continue discussions of lessons learned, progress made and future initiatives. As before, we were particularly interested in discussions of what works versus what does not work, as well as what was planned versus what actually happened. Discussion of the interplay of science operations, technical operations, data management operations, and observatory development is particularly encouragedespecially as it impacts the maximization of science value return. The relationships amongst available funding, delivered capabilities/services, and user expectation management and how that informs observatory operations models were other important discussion topics. Demand for support of time-domain observations is steadily increasing as well, driven by the desire to study rare, random events as well as long-term, synoptic phenomena. Such studies are particularly challenging when they require coordination, often unpredictable, between multiple space and ground-based observatories. While this trend has recently been driven by space-based detections of gamma ray bursts, the startup of ground-based timedomain survey facilities (ramping up to the Large Synoptic Survey Telescope in the second half of this decade) will quickly take this challenge to a new level. Progress reports from new facilities coming online and existing facilities facing major new operational challenges were particularly solicited. 
This volume contains the contributions, both oral and poster, to this conference from the international community.

Alison B. Peck Robert L. Seaman Chris R. Benn 